\title{
Multicast Multigroup Beamforming for Per-antenna Power Constrained Large-scale Arrays
}

\author{
Dimitrios Christopoulos*, Symeon Chatzinotas* and Björn Ottersten* \\ ${ }^{*}$ SnT - securityandtrust.lu, University of Luxembourg \\ email: \{dimitrios.christopoulos, symeon.chatzinotas, bjorn.ottersten\}@uni.lu
}

\begin{abstract}
Large in the number of transmit elements, multiantenna arrays with per-element limitations are in the focus of the present work. In this context, physical layer multigroup multicasting under per-antenna power constrains, is investigated herein. To address this complex optimization problem lowcomplexity alternatives to semi-definite relaxation are proposed. The goal is to optimize the per-antenna power constrained transmitter in a maximum fairness sense, which is formulated as a non-convex quadratically constrained quadratic problem. Therefore, the recently developed tool of feasible point pursuit and successive convex approximation is extended to account for practical per-antenna power constraints. Interestingly, the novel iterative method exhibits not only superior performance in terms of approaching the relaxed upper bound but also a significant complexity reduction, as the dimensions of the optimization variables increase. Consequently, multicast multigroup beamforming for large-scale array transmitters with per-antenna dedicated amplifiers is rendered computationally efficient and accurate. A preliminary performance evaluation in large-scale systems for which the semi-definite relaxation constantly yields non rank-1 solutions is presented.
\end{abstract}

Index Terms-Large-scale Multicasting; Successive Convex Approximation;

\section{INTRODUCTION \& RELATED WORK}

Highly demanding applications (e.g. video broadcasting) stretch the throughput limits of multiuser broadband systems. To provide for such requirements, the adaptation of the physical layer design of next generation multi-antenna wireless communication systems to the needs of the higher network layers is imminent. In this direction, physical layer (PHY) multicasting has the potential to efficiently address the nature of future traffic demand and has become part of the new generation of communication standards. In-line with the recent trends for spectrally efficient massive multiple input multiple output (MIMO) wireless systems [1], the topic of multicasting over large-scale antenna arrays arises. A brief review of the state-of-the art in multicasting follows.

\section{A. PHY Multicasting}

The NP-hard multicast problem was defined and accurately approximated by semi-definite relaxation (SDR) and Gaussian randomization in [2]. Extending the multicast concept, a unified framework for physical layer multicasting to multiple co-channel groups, where independent sets of common data are transmitted to groups of users by the multiple antennas, was given in [3], [4]. In parallel to [3], the work of [5] involved dirty paper coding methods that are bound to increase the complexity of the system. Next, a convex approximation method for the max min fair optimization was proposed in [6], exhibiting increased performance as the number of users per group grows, but for relatively low numbers of transmit antennas. In the same context, a similar iterative convex approximation method, this time for the total power minimization under quality-of-service (QoS) constraints formulation, was considered in [7]. In this case, the conservative convex approximation of [8] was employed and a channel phase based, user scheduling method was performed as a second step towards increasing the tightness of the approximation. Finally, in [9], the multicast multigroup problem, was solved based on approximations and uplink-downlink duality.

The hitherto reviewed literature on multigroup multicast beamforming has only considered sum-power constraints (SPCs) at the transmitter side. Amid this extensive literature, the optimal multigroup multicast precoders when a maximum limit is imposed on the transmitted power of each antenna, have only recently been derived in [10], [11]. Therein, a consolidated solution for the weighted max-min fair multigroup multicast beamforming problem under per-antenna constraints (PACs) is presented. This framework is based on SDR and Gaussian randomization to solve the QoS problem and bisection to derive an accurate approximation of the non-convex max min fair formulation. However, as detailed in [11], the PACs are bound to increase the complexity of the optimization problem and reduce the accuracy of the approximation, especially as the number of transmit antennas is increasing. These observations necessitate the investigation of lower complexity, accurate approximations that can be applied on large-scale antenna arrays, constrained by practical, per-antenna power limitations.

\section{B. Successive Convex Approximation}

Inspired by the recent development of the feasible point pursuit (FPP) successive convex approximation (SCA) of non-convex quadratically constrained quadratic problems (QCQPs), as developed in [12], the present work aims at improving the max min fair solutions of [11]. The FPP - SCA tool has been preferred over other existing approximations (for instance [13]) due to its guaranteed feasibility regardless of the initial state of the iterative optimization [12]. 
The rest of the paper is structured as follows. The generic per-antenna power constrained multicast multigroup system model is presented in Sec. II while the max min problem is formulated and solved in Sec. III. In Sec. IV, the performance of the design is evaluated for a specific system setup. Finally, Sec. V concludes the paper.

Notation: In the remainder of this paper, bold face lower case and upper case characters denote column vectors and matrices, respectively. The operators $(\cdot)^{\dagger},|\cdot|$ and $\otimes$ correspond to the conjugate transpose, the absolute value and the Kronecker product respectively, while $[\cdot]_{i j}$ denotes the $i, j$-th element of a matrix. An identity matrix of $N \times N$ dimensions is denoted as $\mathbf{I}_{N}$ and its $k$-th column as $\mathbf{e}_{k}$. Calligraphic indexed characters denote sets. $\mathbb{R}_{M}^{+}$denotes the set of real positive $M$ dimensional vectors.

\section{System MOdEL}

Assuming a single transmitter, let $N_{t}$ denote the number of transmitting elements and $N_{u}$ the total number of users served. The input-output analytical expression will read as $y_{i}=$ $\mathbf{h}_{i}^{\dagger} \mathbf{x}+n_{i}$, where $\mathbf{h}_{i}^{\dagger}$ is a $1 \times N_{t}$ vector composed of the channel coefficients (i.e. channel gains and phases) between the $i$-th user and the $N_{t}$ antennas of the transmitter, $\mathrm{x}$ is the $N_{t} \times 1$ vector of the transmitted symbols and $n_{i}$ is the independent complex circular symmetric (c.c.s.) independent identically distributed (i.i.d) zero mean Additive White Gaussian Noise (AWGN) measured at the $i$-th user's receive antenna. Focusing on a multigroup multicasting scenario, let there be a total of $1 \leq G \leq N_{u}$ multicast groups with $\mathcal{I}=\left\{\mathcal{G}_{1}, \mathcal{G}_{2}, \ldots \mathcal{G}_{G}\right\}$ the collection of index sets and $\mathcal{G}_{k}$ the set of users that belong to the $k$-th multicast group, $k \in\{1 \ldots G\}$. Each user belongs to only one group, thus $\mathcal{G}_{i} \cap \mathcal{G}_{j}=\varnothing, \forall i, j \in\{1 \cdots G\}$. Let $\mathbf{w}_{k} \in \mathbb{C}^{N_{t} \times 1}$ denote the precoding weight vector applied to the transmit antennas to beamform towards the $k$-th group. The assumption of independent data transmitted to different groups renders the symbol streams $\left\{s_{k}\right\}_{k=1}^{G}$ mutually uncorrelated and the total power radiated from the antenna array is $P_{t o t}=$ $\sum_{k=1}^{G} \mathbf{w}_{k}^{\dagger} \mathbf{w}_{k}$. The power radiated by each antenna element is a linear combination of all precoders $P_{n}=\left[\sum_{k=1}^{G} \mathbf{w}_{k} \mathbf{w}_{k}^{\dagger}\right]_{n n}$, where $n \in\left\{1 \ldots N_{t}\right\}$ is the antenna index.

\section{MulticAst Multigroup Under PACS}

\section{A. SDR Based Solution}

\section{1) Max-Min Fair Formulation:}

\begin{aligned} & $\mathcal{F}: \max _{t,\left\{\mathbf{w}_{k}\right\}_{k=1}^{G}} t \\ &$ subject to $\frac{1}{\gamma_{i}} \frac{\left|\mathbf{w}_{k}^{\dagger} \mathbf{h}_{i}\right|^{2}}{\sum_{l \neq k}\left|\mathbf{w}_{l}^{\dagger} \mathbf{h}_{i}\right|^{2}+\sigma_{i}^{2}} \geq t \\ &, \forall i \in \mathcal{G}_{k}, k, l \in\{1 \ldots G\} \\ &$, and to $\quad {\left[\sum_{k=1}^{G} \mathbf{w}_{k} \mathbf{w}_{k}^{\dagger}\right]_{n n} \leq P_{n} \\ &, \forall n \in\left\{1 \ldots N_{t}\right\} \\ &$, \hline\end{aligned}

where $\mathbf{w}_{k} \in \mathbb{C}^{N_{t}}$ and $t \in \mathbb{R}^{+}$. The notation $\sum_{l \neq k}$ states that aggregate interference from all co-channel groups is calculated. Problem $\mathcal{F}$ receives as inputs the PACs vector $\mathbf{p}=\left[P_{1}, P_{2} \ldots P_{N_{t}}\right]$ and the target SINRs vector $\mathbf{g}=$ $\left[\gamma_{1}, \gamma_{2}, \ldots \gamma_{N_{u}}\right]$. Its goal is to maximize the slack variable $t$ while keeping all SINRs above this value. Thus, it constitutes a max-min problem that guarantees fairness amongst users. The main complication of problem $\mathcal{F}$ lies in constraint (1), where a multiplication of the two optimization variables takes place. To reduce this formulation into the more tractable QCQP form, the following considerations are emanated.

2) Per-antenna Power Minimization: A relation between the fairness and the power minimization problems for the multicast multigroup case under SPCs was firstly established in [4]. As a result, by bisecting the solution of the QoS optimization, a solution to the weighted fairness problem can be derived. Nevertheless, fundamental differences between the $\mathrm{SPC}$ formulation and the PAC problem $\mathcal{F}$, complicate the solution. In more detail, the PACs -i.e (2) - are not necessarily met with equality. A more detailed discussion on this can be found in [11]. Therefore, a per-antenna power minimization problem has been proposed in [11], as

$$
\begin{array}{rl}
\mathcal{Q}: \min _{r,\left\{\mathbf{w}_{k}\right\}_{k=1}^{G}} & r \\
\text { subject to } & \frac{\left|\mathbf{w}_{k}^{\dagger} \mathbf{h}_{i}\right|^{2}}{\sum_{l \neq k}\left|\mathbf{w}_{l}^{\dagger} \mathbf{h}_{i}\right|^{2}+\sigma_{i}^{2}} \geq \gamma_{i}, \\
& \forall i \in \mathcal{G}_{k}, k, l \in\{1 \ldots G\}, \\
\text { and to } \quad \frac{1}{P_{n}}\left[\sum_{k=1}^{G} \mathbf{w}_{k} \mathbf{w}_{k}^{\dagger}\right]_{n n} \leq r, \\
& \forall n \in\left\{1 \ldots N_{t}\right\}, \\
\hline
\end{array}
$$

with $r \in \mathbb{R}^{+}$. Problem $\mathcal{Q}$ receives as input SINR constraints for all users, defined before as $\mathbf{g}$, as well as the per antenna power constraint vector $\mathbf{p}$ of (2). The introduction of the slackvariable $r$, constraints the power consumption of each and every antenna. Subsequently, at the optimum $r^{*}$, the maximum power consumption out of all antennas is minimized and this solution is denoted as $r^{*}=\mathcal{Q}(\mathbf{g}, \mathbf{p})$.

Claim 1: Problems $\mathcal{F}$ and $\mathcal{Q}$ are related as follows

$$
\begin{array}{r}
1=\mathcal{Q}(\mathcal{F}(\mathbf{g}, \mathbf{p}) \cdot \mathbf{g}, \mathbf{p}) \\
t=\mathcal{F}(\mathbf{g}, \mathcal{Q}(t \cdot \mathbf{g}, \mathbf{p}) \cdot \mathbf{p})
\end{array}
$$

(for proof cf. [11])

3) Bisection: The establishment of claim 1 allows for the application of the bisection method, as developed in [2], [4]. The solution of $r^{*}=\mathcal{Q}_{r}\left(\frac{L+U}{2} \mathbf{g}, \mathbf{p}\right)$ is obtained by bisecting the interval $[L, U]$ as defined by the minimum and maximum SINR values. Since $t=(L+U) / 2$ represents the SINR, it will always be positive or zero. Thus, $L=0$. Also, if the system was interference free while all the users had the channel of the best user, then the maximum worst SINR would be attained, thus $U=\max _{i}\left\{P_{t o t} \mathbf{Q}_{i} / \sigma_{i}\right\}$. If $r^{*}<1$, then the lower bound of the interval is updated with this value. Otherwise the value 
is assigned to the upper bound of the interval. Bisection is iteratively performed until an the interval size is reduced to a pre-specified value $\epsilon$ (herein, $\epsilon=10^{-3}$ ). This value needs to be dependent on the magnitude of $L$ and $U$ so that the accuracy of the solution is maintained regardless of the region of operation. After a finite number of iterations, the optimal value of $\mathcal{F}$ is given as the resulting value for which $L$ and $U$ become almost identical, providing an accurate solution for $\mathcal{F}$.

4) Relaxation and Gaussian Randomization: The bisection method, as previously discussed, overcomes the non-convexity due to the multiplication of two variables, namely $t$ and $\mathbf{w}$ in constraint (1). However, problem $\mathcal{Q}$ still remains non-convex. Based on the observation that $\left|\mathbf{w}_{k}^{\dagger} \mathbf{h}_{i}\right|^{2}=\mathbf{w}_{k}^{\dagger} \mathbf{h}_{i} \mathbf{h}_{i}^{\dagger} \mathbf{w}_{k}=$ $\operatorname{Tr}\left(\mathbf{w}_{k}^{\dagger} \mathbf{h}_{i} \mathbf{h}_{i}^{\dagger} \mathbf{w}_{k}\right)=\operatorname{Tr}\left(\mathbf{w}_{k} \mathbf{w}_{k}^{\dagger} \mathbf{h}_{i} \mathbf{h}_{i}^{\dagger}\right)$ and with the change of variables $\mathbf{X}_{i}=\mathbf{w}_{i} \mathbf{w}_{i}^{\dagger}$, one can easily identify that the nonconvexity of $\mathcal{Q}$ lies in the necessity to constrain variable $\mathbf{X}$ to have a unit rank. By dropping this constraint, the non-convex $\mathcal{Q}$ can be relaxed to $\mathcal{Q}_{r}$, which reads as

$$
\begin{aligned}
& \mathcal{Q}_{r}: \min _{r,\left\{\mathbf{X}_{k}\right\}_{k=1}^{G}} r \\
& \text { subject to } \frac{\operatorname{Tr}\left(\mathbf{h}_{i} \mathbf{h}_{i}^{\dagger} \mathbf{X}_{k}\right)}{\sum_{l \neq k} \operatorname{Tr}\left(\mathbf{h}_{i} \mathbf{h}_{i}^{\dagger} \mathbf{X}_{l}\right)+\sigma_{i}^{2}} \geq \gamma_{i}, \\
& \\
& \quad \forall i \in \mathcal{G}_{k}, k, l \in\{1 \ldots G\}, \\
& \text { and to } \frac{1}{P_{n}}\left[\sum_{k=1}^{G} \mathbf{X}_{k}\right]_{n n} \leq r \\
& \\
& \quad \forall n \in\left\{1 \ldots N_{t}\right\}, \\
& \text { and to } \quad \mathbf{X}_{k} \succeq 0, \forall k \in\{1 \ldots G\},
\end{aligned}
$$

Following this relaxation, the derivation of the optimal value $\mathbf{w}^{*}$ requires a rank-1 approximation over $\mathbf{X}^{*}$. The approximation with the highest accuracy is proven to be the Gaussian approximation [14]. In summary, this procedure involves the generation of precoding vectors drawn from a Gaussian distribution with statistics defined by the relaxed solution. After generating a a number of instances and re-scaling them, the solution with the closest performance to the relaxed upper bound, as given by the optimal point of $\mathcal{Q}_{r}$ is chosen. More details on the SDR based solution under PACs, can be found in [11].

\section{B. Successive Convex Approximation}

Problem $\mathcal{Q}$ belongs in the general class of non-convex QCQPs for which the SDR technique is proven to be a powerful and computationally efficient approximation technique [14]. However, the FPP - SCA, a recently proposed alternative to SDR, is herein considered [12]. By defining $\mathbf{w}_{\text {tot }}=\left[\mathbf{w}_{1}^{\dagger}, \mathbf{w}_{2}^{\dagger} \ldots \mathbf{w}_{G}^{\dagger}\right]^{\dagger}$, the $i$-th SINR constraint reads as

$$
\mathbf{w}_{t o t}^{\dagger} \mathbf{A}_{i} \mathbf{w}_{t o t} \leq-\gamma_{i} \sigma_{i}^{2}
$$

where $\mathbf{A}_{i}=\mathbf{A}_{i}^{(+)}+\mathbf{A}_{i}^{(-)}$with $\mathbf{A}_{i}^{(+)}=\gamma_{i}\left(\mathbf{I}_{G}-\operatorname{diag}\left\{\mathbf{e}_{k}\right\}\right) \otimes$ $\mathbf{h}_{i} \mathbf{h}_{i}^{\dagger}$ and $\mathbf{A}_{i}^{(-)}=-\operatorname{diag}\left\{\mathbf{e}_{k}\right\} \otimes \mathbf{h}_{i} \mathbf{h}_{i}^{\dagger}, \forall i \in \mathcal{G}_{k}$. Assuming a random point $\mathbf{z}$, then by the definition of a semi-definite matrix $\mathbf{A}_{i}^{(-)}$we have $\left(\mathbf{w}_{t o t}-\mathbf{z}\right)^{\dagger} \mathbf{A}_{i}^{(-)}\left(\mathbf{w}_{t o t}-\mathbf{z}\right) \leq 0$. By expanding this, a linear restriction of $\mathbf{w}_{\text {tot }}$ around $\mathbf{z}$ reads as

$$
\mathbf{w}_{t o t}^{\dagger} \mathbf{A}_{i}^{(-)} \mathbf{w}_{t o t} \leq 2 \operatorname{Re}\left\{\mathbf{z}^{\dagger} \mathbf{A}_{i}^{(-)} \mathbf{w}_{t o t}\right\}-\mathbf{z}^{\dagger} \mathbf{A}_{i}^{(-)} \mathbf{z} .
$$

Consequently, the SINR constraint (10) can be replaced by

$$
\mathbf{w}_{\text {tot }}^{\dagger} \mathbf{A}_{i}^{(+)} \mathbf{w}_{\text {tot }}+2 \operatorname{Re}\left\{\mathbf{z}^{\dagger} \mathbf{A}_{i}^{(-)} \mathbf{w}_{t o t}\right\}-\mathbf{z}^{\dagger} \mathbf{A}_{i}^{(-)} \mathbf{z} \leq-\gamma_{i} \sigma_{i}^{2},
$$

in which the unknown variables are quadratic over a semidefinite matrix. By adding slack penalties $\mathbf{s} \in \mathbb{R}_{\left(N_{u}+1\right)}^{+}$, the the original QCQP problem $\mathcal{Q}$ can be approximated by

$$
\begin{aligned}
& \mathcal{Q}_{S C A}: \min _{r, \mathbf{w}_{\text {tot }}, \mathbf{s}} r+\lambda\|\mathbf{s}\| \\
& \text { s.t. } \mathbf{w}_{\text {tot }}^{\dagger} \mathbf{A}_{i}^{(+)} \mathbf{w}_{t o t}+2 \operatorname{Re}\left\{\mathbf{z}^{(j) \dagger} \mathbf{A}_{i}^{(-)} \mathbf{w}_{t o t}\right\} \\
&-\mathbf{z}^{(j) \dagger} \mathbf{A}_{i}^{(-)} \mathbf{z}^{(j)} \leq-\gamma_{i} \sigma_{i}^{2}+s_{i} \\
& \forall i \in \mathcal{G}_{k}, k, l \in\{1 \ldots G\}, \\
& \text { and to } \frac{1}{P_{n}}\left[\mathbf{w}_{t o t} \mathbf{w}_{t o t}^{\dagger}\right]_{n n} \leq r+s_{N_{u}+1} \\
& \forall n \in\left\{1 \ldots N_{t}\right\},
\end{aligned}
$$

where $r \in \mathbb{R}^{+}, \lambda \in \mathbb{R}$ is a fixed input parameter and $\mathbf{z}^{(j)}$ is the $j$-th instance of the introduced auxiliary variable. In each instance of the SCA algorithm, $\mathcal{Q}_{S C A}$ is solved and the starting point is updated as $\mathbf{z}^{(j+1)}=\mathbf{w}_{\text {tot }}^{(j)}$. The iterative process is repeated until the guaranteed convergence [12].

\section{Complexity \& Convergence discussions}

An important discussion involves the complexity of the employed techniques to approximate a solution of the highly complex, NP-hard multigroup multicast problem under PACs. Focusing on the SDR based solution of [11], the main complexity burden originates from the relaxed $\mathcal{Q}_{r}$. The total worst case complexity of the SDR based solution of $\mathcal{F}$, as in detail is calculated in [11], is summarised in the following. Initially, a bisection search is performed over $\mathcal{Q}_{r}$ to obtain the relaxed solution. This bisection runs for $N_{i t e r}=\left\lceil\log _{2}\left(U_{1}-L_{1}\right) / \epsilon_{1}\right\rceil$ where $\epsilon_{1}$ is the desired accuracy of the search. Typically $\epsilon_{1}$ needs to be at least three orders of magnitude below the magnitudes of $U_{1}, L_{1}$ for sufficient accuracy. In each iteration of the bisection search, problem $\mathcal{Q}_{r}$ is solved. This SDP has $G$ matrix variables of $N_{t} \times N_{t}$ dimensions and $N_{u}+N_{t}$ linear constraints. Moreover, in each iteration not more than $\mathcal{O}\left(G^{3} N_{t}^{6}+G N_{t}^{3}+N_{u} G N_{t}^{2}\right)$ arithmetic operations will be performed. Next, a fixed number of Gaussian random instances with covariance given by the previous solution are generated. The complexity of this process is linear with respect to the number of Gaussian randomizations. More details on the total complexity of the SDR based algorithm can be found in [11] and are herein omitted for shortness.

As far as the FPP - SCA method is concerned, the iterative process typically runs for a few iterations, especially for larger values of $\lambda$. As in detail explained in [12], convergence is guaranteed. Therein, $\lambda$ was set to 10 while herein even greater values are chosen, i.e. $\lambda=25$ since the optimization problems tackled involve a larger number of constraints. Therefore, 
In each iteration of the FPP - SCA, bisection search is performed over $\mathcal{Q}_{S A C}$. The later, is a second order cone program with a worst case complexity of $\mathcal{O}\left(\left(G N_{t}+N_{u}\right)^{3.5}\right)$. This justifies the user of the FPP - SCA in scenarios with large system dimensions.

\section{Performance Evaluation \& Applications}

\section{A. Uniform Linear Arrays}

To the end of investigating the sensitivity of the proposed algorithm in a generic environment, a uniform linear array (ULA) transmitter is considered. Assuming far-field, line-ofsight conditions, the user channels can be modeled using Vandermonde matrices. For this important special case, the SPC multicast multigroup problem was reformulated into a convex optimization problem and solved in [15], [16]. These results where motivated by the observation that in sum power constrained ULA scenarios, the relaxation consistently yields rank one solutions. Thus, for such cases, the SDR is essentially optimal [2]. Nevertheless, the SDR of the PAC minimization problem in ULAs is not always tight as shown in [11].

Let us consider a ULA serving 4 users allocated to 2 distinct groups. In Fig. 1, its radiation pattern for $N_{t}=8$ antennas and for co-group angular separation $\theta_{a}=35^{\circ}$ is plotted. A total power budget of $P=-3 \mathrm{dBW}$ is equally distributed amongst the available antennas. For the Gaussian randomization, $N_{\text {rand }}=100$ instances are considered. Clearly, the multigroup multicast beamforming optimizes the lobes to reduce interferences between the two groups. The beam patterns from both SDR and FPP - SCA solutions are included in Fig. 1. The superiority in terms of minimum achievable SINR of the latter solution is apparent. Hereafter, the performance evaluation will be based on the minimum user rate, since in the optimization all users are equally weighted.

Firstly, the performance with respect to the angular separation of co-group users is investigated, as $\theta_{a}$ is increased for both groups in the fashion indicated in Fig. 1. In Fig. 2, when co-group users are collocated, i.e. $\theta_{a}=0^{\circ}$, the highest minimum rate is attained. As the separation increases, the rate is reduced reaching a local minimum when interfering users are placed in the same position, i.e. $\theta_{a}=45^{\circ}$. Then, the lowest value is observed when co-group users are orthogonal, i.e. $\theta_{a}=90^{\circ}$. In Fig. 2, the lack of tightness of the relaxation for the SDR based solution is clear as the channel conditions are deteriorating. The only exception is when $\theta_{a}=60^{\circ}$, where the inherent symmetricity of the ULA transmitter is providing sufficient conditions for a rank-1 solution to be easily obtained. Interestingly, this is the only situation where the FPP - SCA method provides a suboptimal solution. For all other instances, the superiority of the lower complexity solution is clear. Consequently, the FPP - SCA outperforms SDR, over the majority of the span of the angular separations, for moderately sized ULAs. In the same setting, the normalized simulation time to compute each precoder is given in Fig. 3. Clearly, when the SDR does not yield rank-1 solutions, the FPP - SCA methods can not only provide more accurate solutions but also

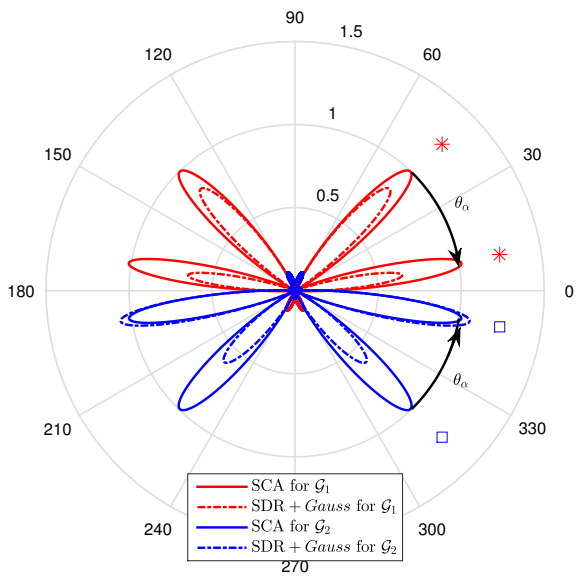

Fig. 1. ULA beampattern for PAC and re-scaled SPC solutions.

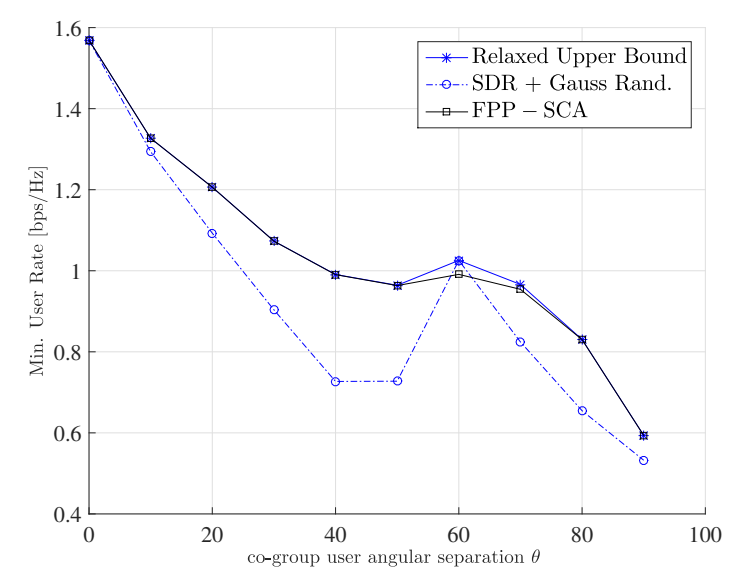

Fig. 2. ULA performance in terms of minimum SINR per group, for increasing co-group user angular separation.

at a significantly reduced time. Almost $50 \%$ of gains in terms of simulation time are observed at $\theta_{a}=80^{\circ}$.

Finally, for an angular separation of $\theta_{a}=60^{\circ}$ where the FPP - SCA solution performs worse, the minimum rate versus an increasing number of transmit antennas is plotted in

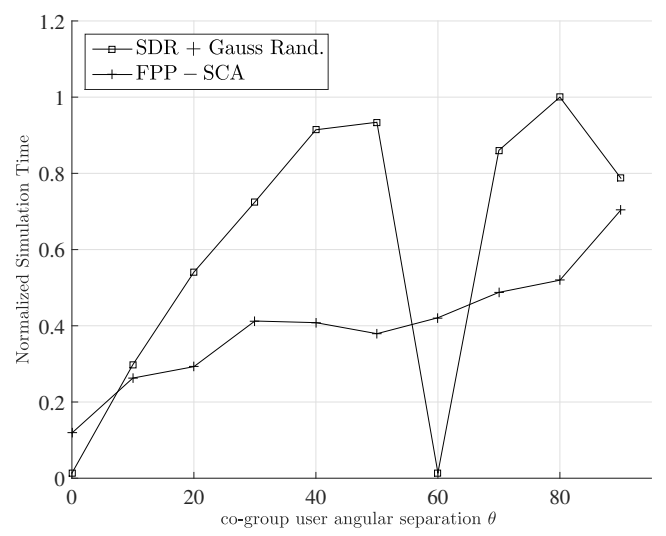

Fig. 3. Normalized simulation time for increasing co-group user angular separation. 


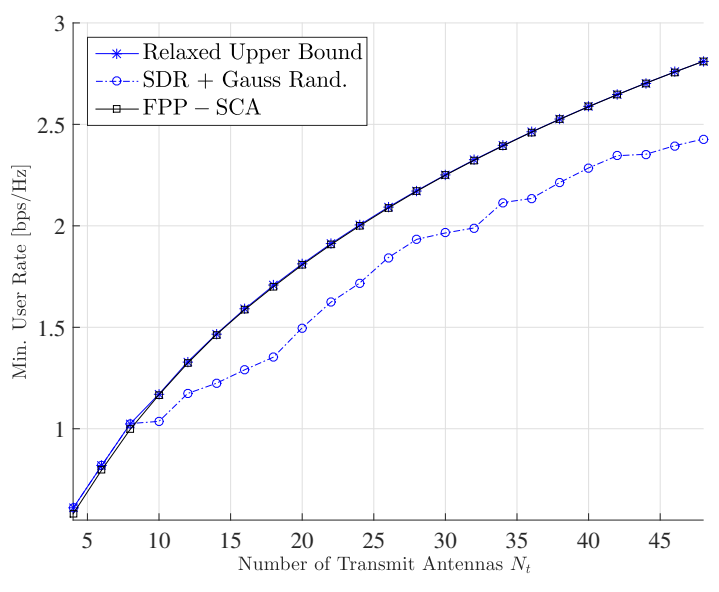

Fig. 4. ULA performance in terms of minimum user rate versus an increasing number of transmit antennas.

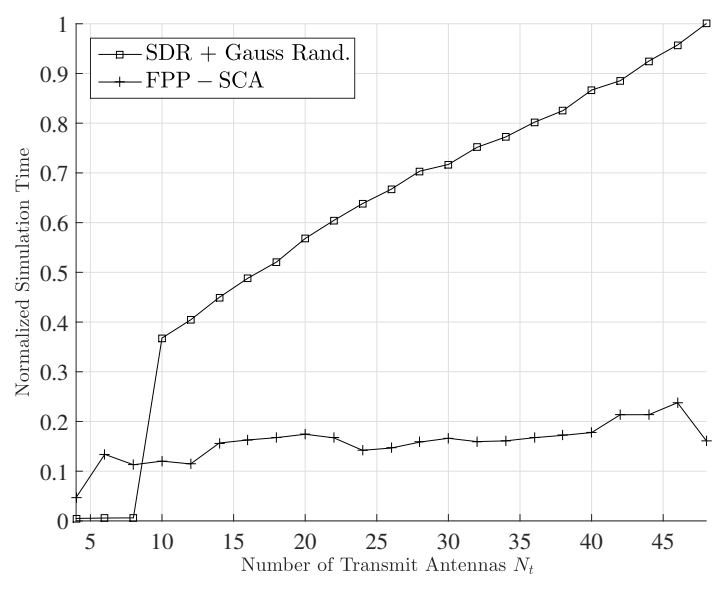

Fig. 5. Normalized simulation time versus an increasing number of transmit antennas.

Fig. 4, while all other simulation parameters remain unaltered. Therein, the benefits of FPP - SCA as the number of antennas is increasing are shown. The SDR solution, fails to provide an accurate solution from 10 antennas onwards. Nevertheless, the FPP - SCA methods provide a tight approximation to the upper bound irrespective of the number of transmit antennas. Impressively, the almost $20 \%$ of performance gains come also at reduced complexity. As shown in Fig. 5, the simulation time can be reduced by even $80 \%$, for large-scale antenna arrays. It should be clarified, that the simulation time figures do not follow the complexity dependence given in Sec. III-C simply because the considerations mentioned therein involve worst case complexity. Existing solvers employed typically exploit the specific structure of matrices thus reducing the actual execution time.

\section{CONCLUSIONS}

Herein, the $\max -\min$ fair multicast multigroup problem under PACs is solved for large-scale antenna arrays. Impressively, the accurate and low complexity FPP - SCA methods outperform existing SDR based approaches both in terms of complexity as well as accuracy, as the number of transmit antennas increases. Future extensions of this work involve different optimization criteria such as the sum rate maximization as well as robust formulations.

Acknowledgements: this work was supported by the following research projects (funding source), SEMIGOD, SATSENT (National Research Fund, Luxembourg), SANSA (European Commission, H2020), PreDem and FGBBF (European Space Agency).

\section{REFERENCES}

[1] F. Rusek, D. Persson, B. K. Lau, E. Larsson, T. Marzetta, O. Edfors, and F. Tufvesson, "Scaling up MIMO: Opportunities and challenges with very large arrays," IEEE Signal Processing Mag., vol. 30, no. 1, pp. 40-60, Jan 2013.

[2] N. Sidiropoulos, T. Davidson, and Z.-Q. Luo, "Transmit beamforming for physical-layer multicasting," IEEE Trans. Signal Process., vol. 54, no. 6, pp. 2239-2251, 2006.

[3] E. Karipidis, N. Sidiropoulos, and Z.-Q. Luo, "Transmit beamforming to multiple co-channel multicast groups," in Proc. of 1st Int. Workshop on Comput. Adv. in Multi-Sensor Adapt. Process. (CAMSAP), 2005, pp. 109-112.

[4] E. Karipidis, N. Sidiropoulos, and Z.-Q. Luo, "Quality of service and max-min fair transmit beamforming to multiple co-channel multicast groups," IEEE Trans. Signal Process., vol. 56, no. 3, pp. 1268-1279, 2008.

[5] Y. Gao and M. Schubert, "Group-oriented beamforming for multistream multicasting based on quality-of-service requirements," in Proc. of 1st Int. Workshop on Comput. Adv. in Multi-Sensor Adapt. Process. (CAMSAP), 2005, pp. 193-196.

[6] A. Schad and M. Pesavento, "Max-min fair transmit beamforming for multi-group multicasting," in Proc. of Int. ITG Workshop on Smart Ant. (WSA), 2012, pp. 115-118.

[7] N. Bornhorst and M. Pesavento, "An iterative convex approximation approach for transmit beamforming in multi-group multicasting," in Signal Processing Advances in Wireless Communications (SPAWC), 2011 IEEE 12th International Workshop on, June 2011, pp. 426-430.

[8] H. Chen, A. Gershman, and S. ShahbazPanahi, "Distributed peer-to-peer beamforming for multiuser relay networks," in Acoustics, Speech and Signal Processing, 2009. ICASSP 2009. IEEE International Conference on, April 2009, pp. 2265-2268.

[9] Y. C. B. Silva and A. Klein, "Linear transmit beamforming techniques for the multigroup multicast scenario," IEEE Trans. Veh. Technol., vol. 58, no. 8, pp. 4353-4367, 2009.

[10] D. Christopoulos, S. Chatzinotas, and B. Ottersten, "Multicast multigroup beamforming under per-antenna power constraints," in Proc. of IEEE International Communications Conference (ICC), Sydney, AU, Jul. 2014, preprint: arXiv:1407.0004 [cs.IT].

[11] D. Christopoulos, S. Chatzinotas, and B. Ottersten, "Weighted fair multicast multigroup beamforming under per-antenna power constraints," vol. 62 , no. 19 , pp. $5132-5142$, Oct. 2014.

[12] O. Mehanna, K. Huang, B. Gopalakrishnan, A. Konar, and N. Sidiropoulos, "Feasible point pursuit and successive approximation of non-convex QCQPs," IEEE Signal Process. Lett., vol. 22, no. 7, pp. 804-808, Jul. 2015.

[13] L.-N. Tran, M. Hanif, and M. Juntti, "A conic quadratic programming approach to physical layer multicasting for large-scale antenna arrays," IEEE Signal Process. Lett., vol. 21, no. 1, pp. 114-117, Jan. 2014.

[14] Z.-Q. Luo, W.-K. Ma, A.-C. So, Y. Ye, and S. Zhang, "Semidefinite relaxation of quadratic optimization problems," IEEE Signal Processing Mag., vol. 27, no. 3, pp. 20-34, 2010.

[15] E. Karipidis, N. Sidiropoulos, and Z.-Q. Luo, "Convex transmit beamforming for downlink multicasting to multiple co-channel groups," in Proc. of IEEE Int. Conf. on Acoustics, Speech and Signal Proc. (ICASSP), vol. 5, May 2006.

[16] E. Karipidis, N. Sidiropoulos, and Z.-Q. Luo, "Far-field multicast beamforming for uniform linear antenna arrays," IEEE Trans. Signal Process., vol. 55, no. 10, pp. 4916-4927, Oct 2007. 